\title{
Investigation of piezomagnetism in nickel ferrite
}

\author{
Alex Aubert*, Vincent Loyau, Frédéric Mazaleyrat, and Martino LoBue \\ SATIE UMR 8029 CNRS, ENS Paris-Saclay, Gif-sur-Yvette, 91190 FRANCE
}

\begin{abstract}
Applications of magnetostrictive materials like sensors and energy harvesting devices generally involve the use of the dynamic deformation, i.e. the piezomagnetic effect, induced by an exciting magnetic field $H_{A C}$. However, this effect is rarely measured directly but commonly approximated by the strain derivative $\partial \lambda / \partial H$, which is deduced from the quasi-static magnetostrictive curve. In this paper, we propose an investigation of the piezomagnetic effect in nickel ferrite by directly measuring the dynamic magnetostriction under various exciting field using a dynamic strain-gauge setup. These values are compared with the strain derivative in order to disclose the limitations thereof. In addition, the magnetoelectric effect is measured in a ferrite/PZT bilayer and compared with calculated magnetoelectric coefficient based on an analytical model. When the dynamic magnetostrictive coefficients are integrated in the calculation, a good accuracy is found with experimental values, whereas it is not the case for calculated coefficient based on the strain derivatives. The findings of the present study highlight the importance of accurately characterizing the piezomagnetic effect in magnetostrictive materials in order to optimize their performances in dynamic applications.
\end{abstract}

\section{Index Terms}

Magnetostriction, Piezomagnetism, Magnetoelectric composite, Strain derivative, Nickel ferrite

\section{INTRODUCTION}

M AGNETOSTRICTION is defined as the change in length of a material under the application of a static magnetic field. This property is usually characterized with the classical strain gauge method, which measures a static deformation as function of a polarizing field $H_{D C}$, giving the well-known magnetostriction coefficient $\lambda$ [1]. Today, magnetostrictive materials are used in actuators [2], [3], sonar transducers [4], [5], and sensors [6]. However, for most of these applications, the key feature is not the static magnetostriction but the dynamic one, also known as piezomagnetic effect. Strictly speaking, the piezomagnetic coefficient is defined as the strain oscillation induced by an alternating magnetic field $H_{A C}$ at any bias field $H_{D C}$ [1], [7], [8], which can be written:

$$
q^{A C}=\left(\frac{\partial \lambda_{A C}}{\partial H_{A C}}\right)_{H_{D C}}
$$

with $\lambda_{A C}$ the dynamic magnetostriction and $q^{A C}$ the piezomagnetic coefficient. Nonetheless, today, the piezomagnetic coefficient is usually approximated to the strain derivative of the static magnetostrictive curve:

$$
q^{D C}=\frac{\partial \lambda}{\partial H_{D C}}
$$

with $\lambda$ the static magnetostriction coefficient and $q^{D C}$ the strain derivative. We have recently shown that this approximation may lead into quite relevant errors when evaluaiting ME devices performances particularly when semi-hard magnetostrictive materials (e.g. cobalt ferrites) are involved [8]. In fact, the strain derivative depends on the differential susceptibility $\chi_{\text {diff }}$ whereas the piezomagnetic effect arises from the dynamic susceptibility $\chi_{A C}$ at any bias field. For low susceptibility $(\approx 10)$ semi-hard materials, the dynamic susceptibility is much lower than the differential one, which makes the dynamic deformation (i.e. the piezomagnetic coefficient) much lower than the strain derivative at low exciting field, hence overruling the approximation for such materials.

In case of soft (nickel) ferrites, the permeability is one order of magnitude higher than for semi-hard (cobalt) ferrites. Thus, one might expect the strain derivative to be a reasonable approximation to the piezomagnetic effect at low exciting fields. However, in this case too, the approximation can be rather misleading under different exciting fields due to the high permeability coupled with a quite low saturation magnetostriction characterizing these materials [9].

In the past, piezomagnetic measurement has been investigated in magnetostrictive Terfenol-D alloys to show their potential as transducers. These measurements were performed either with a strain gauge [10]-[12], an accelerometer [7], a laserDopplervibrometry system [13], [14] or by using a three-terminal capacitance technique [15]. More recently, this property was also studied to determine its influence in the magnetoelectric effect, as shown in the case of cobalt ferrite [8] and $\mathrm{SmFe}_{2}$ [16]. To the best of our knowledge, the piezomagnetic effect has never been measured experimentally for nickel ferrite material, which motivates this study. The use of strain gauges for such purpose is optimal, as they allow high mechanical coupling, do not induce

*Present address: Functional Materials, Department of Material Science, Technische Universitat Darmstadt, 64287, Darmstadt, Germany. 
prestress in the measured system and have a high sensitivity [8]. Moreover, there is a growing interest in mechanically coupling magnetostrictive materials, and in particular nickel ferrite, with piezoelectric ones to make magnetoelectric composites [17]. Afterwards, these composites can be integrated in devices to be used as current sensors [18], magnetic field sensors [19], gyrators, inductors [20] and other power electronics applications [21]-[23]. This entails an increasing effort in developing accurate material and device models [24]-[26] and makes the identification of reliable characterization methods an objective of the utmost relevance.

The main purpose of this paper is to show that the commonly used static characterization methods fall short of the aforementioned need of accurate characterization protocols and that full dynamic characterization - even in the case of composites using soft ferrites - is necessary to properly assess the theoretical models and to optimize the composite's performances.

\section{EXPERIMENTAL DETAILS}

Nickel ferrite $\left(\mathrm{NiFe}_{2} \mathrm{O}_{4}\right)$ are produced by mixing nanosized $(<50 \mathrm{~nm})$ oxides $\mathrm{NiO}$ and $\mathrm{Fe}_{2} \mathrm{O}_{3}$ (Sigma-Aldrich) in accurate molar ratio. Powders are mixed in a planetary ball milling during $30 \mathrm{~min}$ at $400 \mathrm{rpm}$, and then ground during $1 \mathrm{~h}$ at $600 \mathrm{rpm}$. The oxide mixture is then processed by Spark Plasma Sintering (SPS) technique where both the reaction and the sintering are performed (reactive sintering). SPS allows to obtain high density samples with nanosized grains in a very short processing time $(<30 \mathrm{~min})$ [27], [28]. The process is performed at $100 \mathrm{MPa}$ and the sample is heated to $550{ }^{\circ} \mathrm{C}$ in $3 \mathrm{~min}$ and kept 5 min at this stage to achieve the reaction. The heating is then increased to $850{ }^{\circ} \mathrm{C}$ in 2 min, and left 3 min at this temperature to do the sintering. Afterwards, the sample is cooled in $10 \mathrm{~min}$. Eventually, the sample is fully re-oxidized by means of an annealing at $1000{ }^{\circ} \mathrm{C}$ during $1 \mathrm{~h}$ in air. The final sample is a disk of $10 \mathrm{~mm}$ in diameter and $2 \mathrm{~mm}$ in thickness with high relative density (97\%), and will be referred as NFO in the following. The magnetoelectric composite is made by bonding the obtained NFO with a commercial PZT disk (Ferroperm PZ27, diameter: $10 \mathrm{~mm}$, thickness: $1 \mathrm{~mm}$ ) using silver epoxy (Epotek E4110), getting a final ME bilayer with thickness of $3 \mathrm{~mm}$ and a diameter of $10 \mathrm{~mm}$. The piezoelectric sample is polarized along the thickness direction.

Static magnetostriction measurements are performed on the nickel ferrite integrated in the ME bilayer using the usual strain gauge method. This measurement is done on the bilayer in order to take into account the prestress induced by the PZT layer on nickel ferrite [29]. The gauge is bonded on the free surface of the ferrite layer (see Fig. 1). The gauge used is a selftemperature-compensated semiconductor gauge (Kyowa KSN-2-120-E4-11). The dynamic magnetostriction measurements are performed using the same gauge and the voltage at its terminal is measured by a lock-in amplifier (EG\&G Princeton 5210). The measurement is performed at low frequency $(80 \mathrm{~Hz})$ to avoid any mechanical resonance phenomena and other parasitic electric signals. The amplitude of the exciting field $H_{A C}$ is varied from $0.08 \mathrm{kA} / \mathrm{m}$ to $25.5 \mathrm{kA} / \mathrm{m}$ in order to highlight its influence in the piezomagnetic coefficient of soft ferrites. Measurements are performed on disks, thus giving the longitudinal and transverse piezomagnetic coefficient, respectively $q_{11}^{A C}$ and $q_{21}^{A C}$, at any given bias field. More details of our experimental setup are given in a previous study [8]. Finally, transverse magnetoelectric measurements $\left(\alpha_{31}\right)$ are performed by measuring the piezoelectric voltage by means of a lock-in amplifier (EG\&G Princeton 5210) with high input impedance (100 M $\Omega$ ). It is worth noting that the lock-in amplifier only measures the fundamental frequency of the signal here.

\section{RESULTS AND DISCUSSION}

Magnetostriction measurements are performed with the magnetic field applied in the radial direction of the disk, either parallel $\left(\lambda_{11}\right)$ or perpendicular $\left(\lambda_{21}\right)$ to the gauge. The results are presented in Fig. 1. The longitudinal magnetostriction saturation is

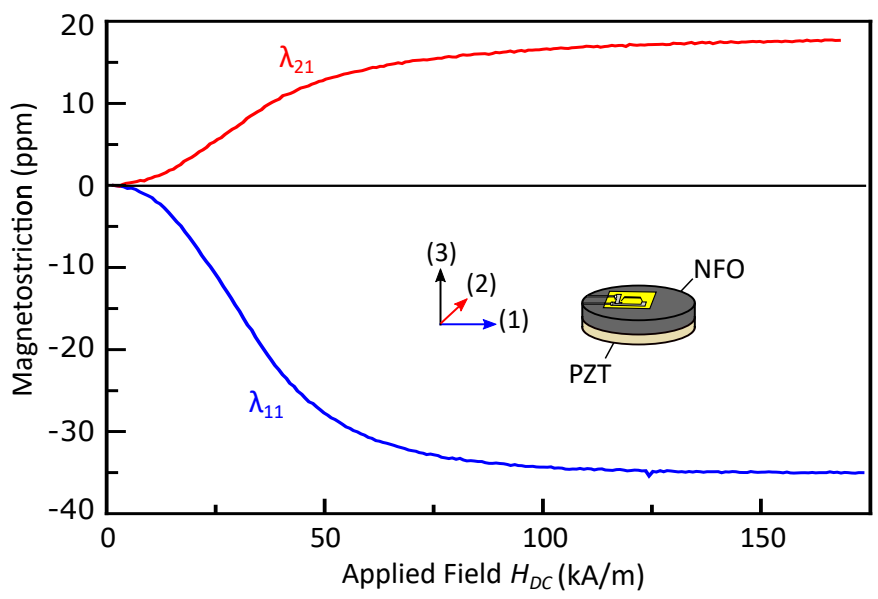

Fig. 1. Longitudinal $\left(\lambda_{11}\right)$ and transverse $\left(\lambda_{21}\right)$ magnetostriction of $\mathrm{NiFe}_{2} \mathrm{O}_{4}$. 


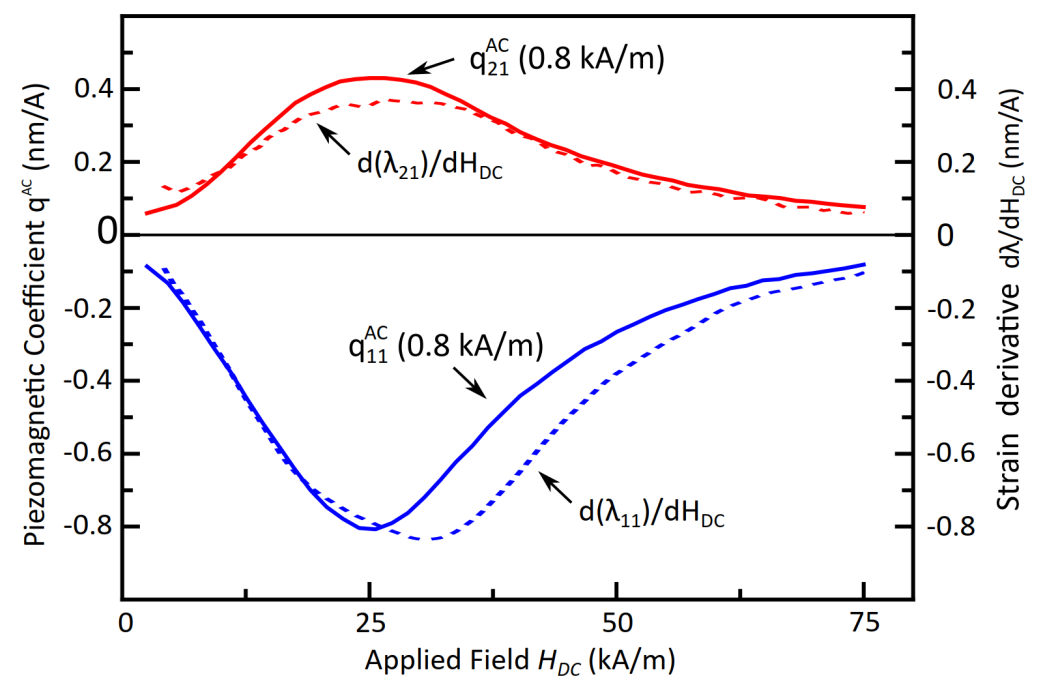

Fig. 2. Strain derivatives (dotted line) of $\mathrm{NiFe}_{2} \mathrm{O}_{4}$ for longitudinal $\mathrm{d}\left(\lambda_{11}\right) / \mathrm{d} H_{D C}$ and transverse $\mathrm{d}\left(\lambda_{21}\right) / \mathrm{d} H_{D C}$ direction. Longitudinal ( $\left.q_{11}^{A C}\right)$ and transverse $\left(q_{21}^{A C}\right.$ ) piezomagnetic coefficient (continuous line) measured with an exciting field $H_{A C}=0.8 \mathrm{kA} / \mathrm{m}$ at $80 \mathrm{~Hz}$ as function of the bias field $H_{D C}$.

$-35 \mathrm{ppm}$ while the transverse one is $18 \mathrm{ppm}$. This gives a longitudinal to transverse ratio of about 2:1 at saturation, which is the expected ratio for isotropic samples [28], [30].The strain derivatives $\mathrm{d} \lambda / \mathrm{d} H_{D C}$ of these magnetostrictive curves were calculated and are plotted in dotted line in Fig. 2. The maximum values obtained are -0.83 and $0.38 \mathrm{~nm} / \mathrm{A}$ for longitudinal and transverse coefficients, respectively. As it was already established from previous studies [30], isotropic samples provide a relation between longitudinal and transverse strain derivative of $\mathrm{d}\left(\lambda_{11}\right) / \mathrm{d} H_{D C} \simeq-2 \mathrm{~d}\left(\lambda_{21}\right) / \mathrm{d} H_{D C}$.

In order to compare these values with piezomagnetic coefficients, the dynamic magnetostriction of the sample was also measured. First, the measurement is performed by applying a low exciting magnetic field of $0.8 \mathrm{kA} / \mathrm{m}(\sim 1 \mathrm{mT})$, which is a typical amplitude used for magnetoelectric characterization and application [9], [17], [29]. The longitudinal and transverse piezomagnetic coefficients measured as function of the bias field are plotted in Fig. 2 and the maximum values obtained are -0.81 and $0.41 \mathrm{~nm} / \mathrm{A}$, respectively. Even though we observe a slight discrepancy in the polarizing field necessary to reach the maximum coefficient, the measurements confirm that strain derivative could be approximated to the piezomagnetic coefficient when excited at low amplitude of magnetic field (approx. $1 \mathrm{mT}$ ). In fact, the relative error of the maximum value in the longitudinal direction is of $2.5 \%$. Regarding the transverse measurement, the approximation is also consistent although we observe a slightly higher value in the case of the piezomagnetic coefficient. As measurements are done far from mechanical resonance, the strain derivative can be expected to represent the upper limit of the piezomagnetic coefficient. Here, we may expect a slight overestimation of the transverse piezomagnetic value due to the weak amplitude (approx. tens of $\mu \mathrm{V}$ ) of the signal measured to obtain $q_{21}^{A C}$, which makes it more sensitive to ambient noise.

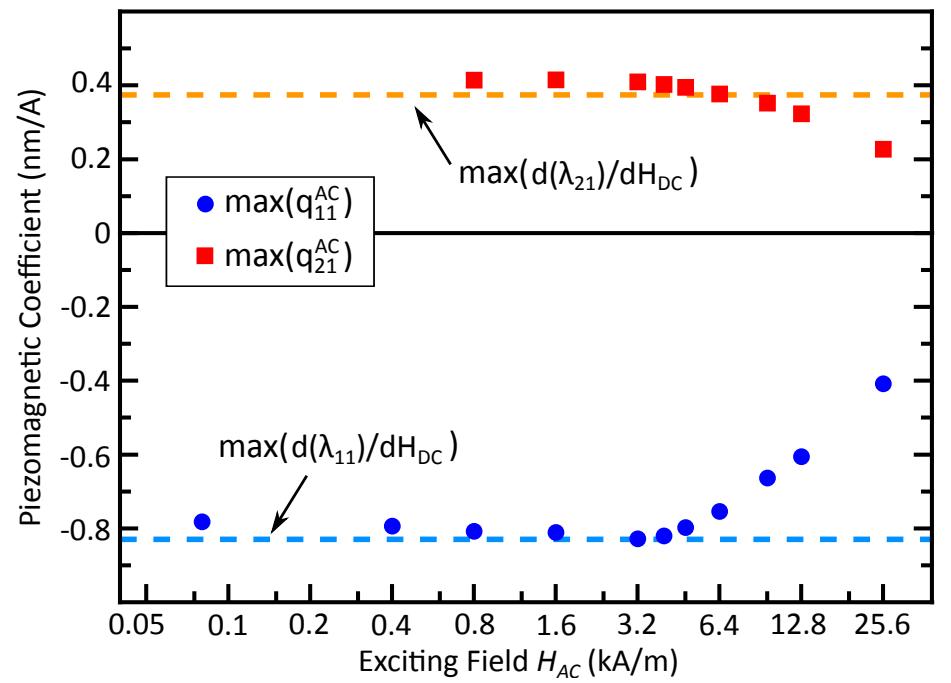

Fig. 3. Longitudinal (circles) and transverse (squares) piezomagnetic coefficient measured for $\mathrm{NiFe}_{2} \mathrm{O}_{4}$ as function of the exciting field $H_{A C}$ at $80 \mathrm{~Hz}$ (semi- $\log _{2}$ plot) and at the optimum bias field $H_{D C}$. The strain derivatives are plotted in dotted line for comparison. 
To further characterize the dynamic deformation of nickel ferrite, we performed dynamic magnetostriction measurements for various amplitudes of the exciting field $H_{A C}: 0.08 \mathrm{kA} / \mathrm{m}, 0.4 \mathrm{kA} / \mathrm{m}, 0.8 \mathrm{kA} / \mathrm{m}, 1.6 \mathrm{kA} / \mathrm{m}, 3.2 \mathrm{kA} / \mathrm{m}, 4 \mathrm{kA} / \mathrm{m}, 4.8 \mathrm{kA} / \mathrm{m}$, $6.4 \mathrm{kA} / \mathrm{m}, 9.5 \mathrm{kA} / \mathrm{m}, 12.7 \mathrm{kA} / \mathrm{m}$ and $25.5 \mathrm{kA} / \mathrm{m}$. The transverse measurement is only possible for amplitudes higher than $0.8 \mathrm{kA} / \mathrm{m}$. In Fig. 3, we plot the maximum piezomagnetic coefficient for the longitudinal and transverse direction. The results show that the piezomagnetic coefficients are, in both directions, constant between $0.08 \mathrm{kA} / \mathrm{m}$ and $3.2 \mathrm{kA} / \mathrm{m}$. It demonstrates that nickel ferrite, contrary to harder magnetic materials as cobalt ferrite [8], has excellent dynamic performances at low exciting field. Another noticeable difference between these two materials is that the dynamic coefficients of nickel ferrite are able to reach the strain derivative values, whereas cobalt ferrite's dynamic coefficient can hardly exceed over $70 \%$ of the quasi-static coefficient when excited at the optimum exciting field [8]. However, as observed in Fig. 3, nickel ferrite reaches an optimum dynamic values around $3.2 \mathrm{kA} / \mathrm{m}$ and then the coefficient in both directions drastically decrease by a factor two at $25.5 \mathrm{kA} / \mathrm{m}$. This, as it will be demonstrated in the next paragraph, results from the non-linearity of the magnetostrictive curve when the material is excited at high field. Thus, the contribution of harmonics increases and the signal of the fundamental frequency which is measured here - decreases.

In order to prove that the dynamic characterization of nickel ferrite is essential to correctly describe its behavior in dynamic applications such as ME sensors, the magnetoelectric effect has first been measured on a NFO/PZT bilayer for various exciting field $H_{A C}$ and as function of the bias field $H_{D C}$. The maximum magnetoelectric coefficients are represented in Fig. 4 (circles). At very low AC field, due to hysteresis (i.e. domain wall pinning), the deformation for a given AC field is not optimum and the magnetoelectric coefficient $\alpha_{31}^{e x p}$ shows a constant value (148 mv/A) until $0.8 \mathrm{kA} / \mathrm{m}$. By further increasing the AC field, an optimum value of $175 \mathrm{mV} / \mathrm{Ais}$ reached at $4 \mathrm{kA} / \mathrm{m}$ before decreasing drastically to $74 \mathrm{mV} / \mathrm{A}$ at $25.5 \mathrm{kA} / \mathrm{m}$. To understand this decrease, the total harmonic distortion $\left(\mathrm{THD}_{\mathrm{F}}\right)$ calculated from the magnetoelectric voltage as function of $H_{A C}$ is also plotted in Fig. 4 (diamond). It appears that the $\mathrm{THD}_{\mathrm{F}}$ increases with increasing $H_{A C}$ because the AC field is so high that the magnetostriction reaches the non-linearity of the magnetostrictive curve [8], [31], [32]. The ME drop is linked to the decrease observed in the piezomagnetic coefficient (see Fig.3) which mainly depends on the permeability of the material as well as its saturation magnetostriction [8], [31], [32]. As nickel ferrite exhibits low longitudinal saturation magnetostriction (-35 ppm) coupled with high permeability $(>50)$, its $\mathrm{THD}_{\mathrm{F}}$ increases rapidly for moderate values of exciting field $(>6.4 \mathrm{kA} / \mathrm{m})$.

Then, we compare the experimentally measured ME values with theoretical ones, which are calculated based on a model that takes into account the piezomagnetic coefficients measured with our dynamic strain gauge setup. The ME coefficient can be expressed by the following formula [17], [29]:

$$
\alpha_{31}=\frac{\eta\left(q_{11}^{A C}+q_{21}^{A C}\right) d_{31}^{e}}{\epsilon_{33}\left[\left(s_{11}^{e}+s_{21}^{e}\right)+\eta \gamma\left(s_{11}^{m}+s_{21}^{m}\right)\right]-2\left(d_{31}^{e}\right)^{2}}
$$

where $\eta$ is the mechanical coupling factor, $d_{31}^{e}$ is the transverse piezoelectric coefficient, $\epsilon_{33}$ is the dielectric permittivity, $s_{i j}$ are the compliances, and $\gamma=\frac{\nu_{e}}{\nu_{m}}=\frac{t_{e}}{t_{m}}$ the volume ratio, with $t_{e}$ and $t_{m}$ the thicknesses of PZT and CFO respectively. The following parameters are used for the calculation: $d_{31}^{e}=-170 \mathrm{pC} / \mathrm{N}, s_{11}^{e}=17 \mathrm{pm}^{2} / \mathrm{N}, s_{21}^{e}=-6.6 \mathrm{pm}^{2} / \mathrm{N}, \epsilon_{r 33}=1800$, $s_{11}^{m}=6.47 \mathrm{pm}^{2} / \mathrm{N}, s_{21}^{m}=-1.84 \mathrm{pm}^{2} / \mathrm{N}$ [17], [29]. The mechanical coupling factor $\eta$ is set to 0.28 , which is in agreement with FEM simulation for a bilayer [8]. The calculated values $\alpha_{31}^{t h}\left(q^{A C}\right)$ which integrate previously estimated piezomagnetic coefficients $q_{11}^{A C}$ and $q_{21}^{A C}$ are plotted in Fig. 4 (squares). The accuracy between experimental and theoretical values is good at low and high exciting field, but a slight discrepancy is observed at the optimum exciting field range $\left(4<H_{A C}<6.4 \mathrm{kA} / \mathrm{m}\right)$. This can be due to the overestimation of the $q_{21}^{A C}$, as discussed previously, which leads to an underestimation of $\alpha_{31}^{t h}\left(q^{A C}\right)$

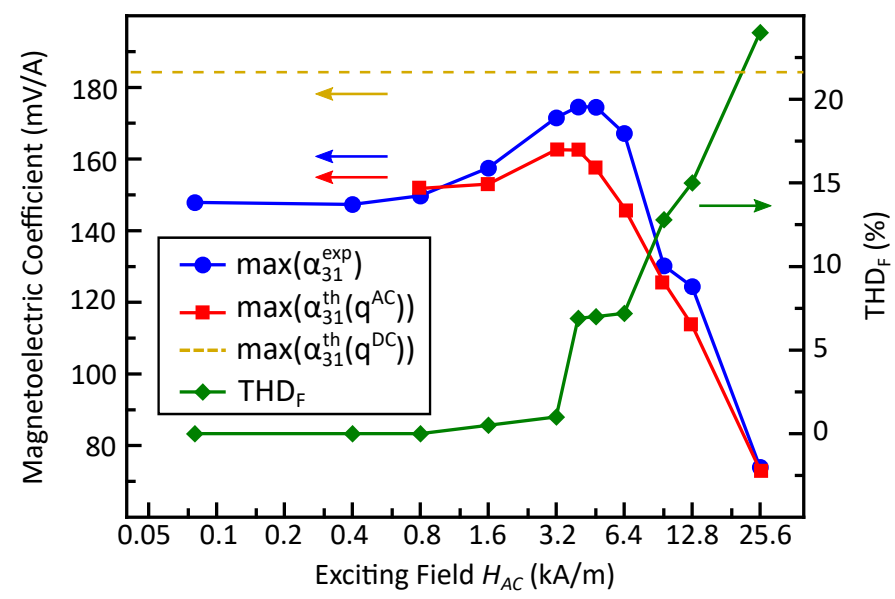

Fig. 4. Maximum magnetoelectric coefficient measured for the ME bilayer NFO/PZT (circles) as function of the exciting field $H_{A C}$ at $80 \mathrm{~Hz}$ (semi-log 2 plot) and at the optimum bias field $H_{D C}$. The theoretical magnetoelectric coefficient calculated from the piezomagnetic curves is plotted in squares and the one based on the strain derivatives in dotted. The total harmonic distortion $\left(\mathrm{THD}_{\mathrm{F}}\right)$ of the magnetoelectric voltage is plotted in diamond. 


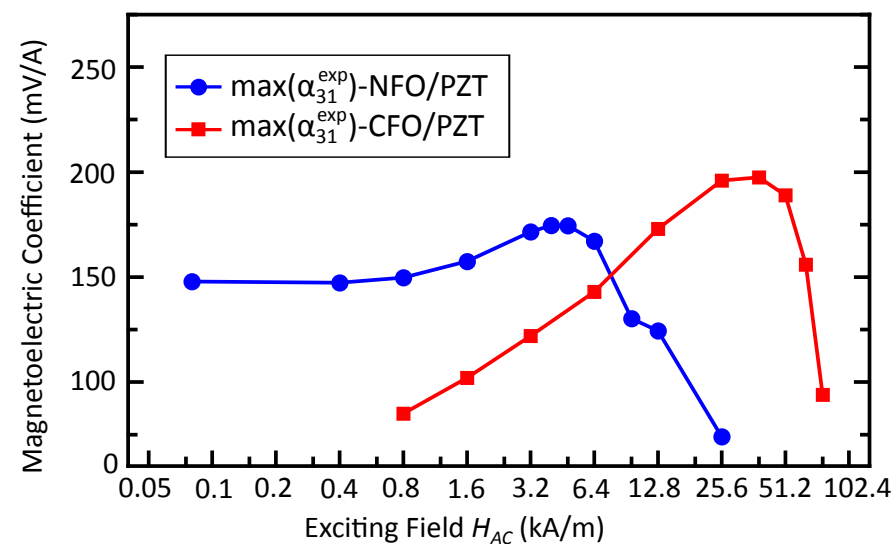

Fig. 5. Maximum magnetoelectric coefficient measured for NFO/PZT (circles) and CFO/PZT (squares) as function of the exciting field $H_{A C}$ at $80 \mathrm{~Hz}$ and at the optimum bias field $H_{D C}$. Values for CFO/PZT are taken from Aubert et al. and refers to the isotropic cobalt ferrite [8].

compared to the $\alpha_{31}^{e x p}\left(q^{A C}\right)$. On the other hand, when the strain derivatives are integrated in the analytical model, the ME coefficient calculated $\alpha_{31}^{t h}\left(q^{D C}\right)$ overestimates the ME effect (see the dotted line in Fig. 4). This demonstrates the necessity to characterize the dynamic magnetostrictive coefficient in order to correctly and fully describe the magnetoelectric one, even in soft ferrites.

Finally, we compare the magnetoelectric results for nickel ferrite (NFO) with the previously reported results for isotropic cobalt ferrite (CFO) [8]. In both cases, the ME composites have the same geometrical aspect ratio. Hence, any differences should mainly be attributed to the dynamic magnetostrictive behavior. The maximum magnetoelectric coefficient (at the optimum $H_{D C}$ ) measured for these bilayers are presented in Fig. 5 as function of the exciting field $H_{A C}$. It appears that ME composites based on cobalt ferrite can reach better magnetoelectric conversion, due to higher dynamic deformation when excited at the optimum field. However, it requires high exciting field to reach the best magnetoelectric coefficient, which cannot be suitable for all dynamic applications. Nickel ferrite has the advantage of being 1.5 times more efficient than cobalt ferrite at low applied fields $H_{A C}(<6.4 \mathrm{kA} / \mathrm{m})$, which are more common for applications. This explains why this material is preferred in magnetoelectric composites. It also justify the better magnetoelectric effect reported in the literature for ME composites integrating NFO, whereas its strain derivative is lower than CFO [33], [34]. This result shows that nickel and cobalt ferrites are complementary due to their differences in magnetic anisotropy and permeability. Either of them can be the more suitable material depending on the field intensity involved in the aimed application.

\section{CONCLUSION}

In summary, we proposed a characterization of the piezomagnetic effect of nickel ferrite, which is of interest for dynamic magnetostrictive sensor applications. It is shown that the dynamic deformation depends on the amplitude of the exciting field and is particularly efficient at low amplitudes $(<4 \mathrm{kA} / \mathrm{m})$ for soft nickel ferrite. It is demonstrated that piezomagnetic coefficients obtained by the dynamic strain gauge setup allow to give an accurate description of the dynamic behaviour unlike the strain derivative. These results are confirmed with magnetoelectric measurements performed on a bilayer NFO/PZT. This study reveals that, even in magnetically soft materials, dynamic characterization is the most reliable method to properly describe magnetostrictives properties, and consequently to correctly assess the performance of the material for dynamic applications. 


\section{REFERENCES}

[1] E. Du Trémolet de Lacheisserie, Magnetostriction, Theory and Applications of Magnetoelasticity. Boca Raton, FL, USA:CRC Press, 1993.

[2] M. Anjanappa and Y. Wu, "Magnetostrictive particulate actuators: configuration, modeling and characterization," Smart Mater. Struct., vol. 6, no. 4, p. 393, 1997. [Online]. Available: http://stacks.iop.org/0964-1726/6/i=4/a=002

[3] S. Karunanidhi and M. Singaperumal, "Design, analysis and simulation of magnetostrictive actuator and its application to high dynamic servo valve," Sensors Actuators A, vol. 157, no. 2, pp. 185 - 197, 2010. [Online]. Available: http://www.sciencedirect.com/science/article/pii/S0924424709004865

[4] C. van der Burgt and A. Stuijts, "Developments in ferrite ceramics with strong piezomagnetic coupling," Ultrasonics, vol. 1, no. 4, pp. 199 - 210, 1963. [Online]. Available: http://www.sciencedirect.com/science/article/pii/0041624X63901689

[5] Y. Kikuchi, "Magnetostrictive materials and applications," IEEE Trans. Magn., vol. 4, no. 2, pp. 107-117, June 1968.

[6] A. Olabi and A. Grunwald, "Design and application of magnetostrictive materials," Mater. Des., vol. 29, no. 2, pp. 469 - 483, 2008. [Online]. Available: http://www.sciencedirect.com/science/article/pii/S0261306907000027

[7] F. Claeyssen, D. Colombani, A. Tessereau, and B. Ducros, "Giant dynamic magnetostrain in rare earth-iron magnetostrictive materials," IEEE Trans. Magn., vol. 27, no. 6, pp. 5343-5345, Nov 1991.

[8] A. Aubert, V. Loyau, Y. Pascal, F. Mazaleyrat, and M. LoBue, "Dynamic magnetostriction of cofe $2 \mathrm{O}_{4}$ and its role in magnetoelectric composites," Phys. Rev. Applied, vol. 9, p. 044035, Apr 2018. [Online]. Available: https://link.aps.org/doi/10.1103/PhysRevApplied.9.044035

[9] V. Loyau, V. Morin, J. Fortineau, M. LoBue, and F. Mazaleyrat, "A method to decrease the harmonic distortion in Mn-Zn ferrite/PZT and Ni-Zn ferrite/PZT layered composite rings exhibiting high magnetoelectric effects," J. Appl. Phys., vol. 118, no. 15, 2015. [Online]. Available: http://scitation.aip.org/content/aip/journal/jap/118/15/10.1063/1.4933266

[10] J. Hudson, S. Busbridge, and A. Piercy, "Dynamic magneto-mechanical properties of epoxy-bonded terfenol-d composites," Sensors Actuators A, vol. 81, no. 1, pp. 294 - 296, 2000. [Online]. Available: http://www.sciencedirect.com/science/article/pii/S0924424799001788

[11] S. W. Or, N. Nersessian, G. P. McKnight, and G. P. Carman, "Dynamic magnetomechanical properties of [112]-oriented terfenol-d/epoxy 1-3 magnetostrictive particulate composites," J. Appl. Phys., vol. 93, no. 10, pp. 8510-8512, 2003. [Online]. Available: http://dx.doi.org/10.1063/1.1555979

[12] S. W. Or, N. Nersessian, and G. P. Carman, "Dynamic magnetomechanical behavior of terfenol-d/epoxy 1-3 particulate composites," IEEE Trans. Magn., vol. 40, no. 1, pp. 71-77, Jan 2004. [Online]. Available: https://doi.org/10.1109/TMAG.2003.821123

[13] C. Lu, P. Li, Y. Wen, A. Yang, C. Yang, J. Yang, W. He, J. Zhang, and W. Li, "Dynamic magnetostrictive properties of magnetization-graded ferromagnetic material and application in magnetoelectric composite," J. Appl. Phys., vol. 115, no. 17, p. 17C726, 2014. [Online]. Available: http://dx.doi.org/10.1063/1.4866089

[14] L. Bian, Y. Wen, and P. Li, "Dynamic magnetomechanical behavior of tbxdy1-x2013;xfey alloy under small-signal ac drive fields superposed with various bias fields," IEEE Trans. Magn., vol. 52, no. 8, pp. 1-5, Aug 2016

[15] B. Kundys, Y. Bukhantsev, S. Vasiliev, D. Kundys, M. Berkowski, and V. P. Dyakonov, "Three terminal capacitance technique for magnetostriction and thermal expansion measurements," Rev. Sci. Instrum., vol. 75, no. 6, pp. 2192-2196, 2004.

[16] Zhang, Jitao, Zhu, Weiwei, Chen, Dongyu, Li, Kang, Zhang, Qingfang, Wang, Xiaolei, Zheng, Xiaowan, Jiang, Liying, and Cao, Lingzhi, "Effects of remanent magnetization on dynamic magnetomechanical and magnetic-sensing characteristics in bi-layer multiferroics," Eur. Phys. J. Appl. Phys., vol. 85, no. 2, p. 20601, 2019. [Online]. Available: https://doi.org/10.1051/epjap/2019180168

[17] V. Loyau, A. Aubert, M. LoBue, and F. Mazaleyrat, "Analytical modeling of demagnetizing effect in magnetoelectric ferrite/PZT/ferrite trilayers taking into account a mechanical coupling," J. Magn. Magn. Mater, vol. 426, pp. 530 - 539, 2017. [Online]. Available: http://www.sciencedirect.com/science/article/pii/S0304885316319436

[18] A. Aubert, V. Loyau, G. Chaplier, F. Mazaleyrat, and M. LoBue, "Enhanced magnetoelectric voltage in ferrite/PZT/ferrite composite for AC current sensor application," J Mater Sci: Mater Electron, vol. 29, p. 14435-14444, 2018. [Online]. Available: https://doi.org/10.1007/s10854-018-9576-0

[19] Y. Ren, J. Ouyang, W. Wang, X. Wu, X. Yang, Y. Zhang, and S. Chen, "Rotating magnetoelectric sensor for dc magnetic field measurement," IEEE Trans. Magn., vol. 54, no. 11, pp. 1-3, Nov 2018.

[20] J. Zhang, D. Chen, D. A. Filippov, Q. Zhang, K. Li, X. Hang, B. Ge, L. Cao, and G. Srinivasan, "Improved tunability in metglas/ferrite/pzt magnetoelectric tunable inductors," IEEE Trans. Magn., vol. 55, no. 7, pp. 1-4, July 2019.

[21] M. Zhu, T. Nan, M. Liu, W. Ren, Z. Zhou, and N. X. Sun, "Voltage tuning of ferromagnetic resonance and linewidth in spinel ferrite/ferroelectric multiferroic heterostructures," IEEE Magnetics Letters, vol. 6, pp. 1-4, 2015.

[22] L. Chen and Y. Wang, "Enhanced magnetic field sensitivity in magnetoelectric composite based on positive magnetostrictive/negative magnetostrictive/piezoelectric laminate heterostructure," IEEE Trans. Magn., vol. 53, no. 11, pp. 1-5, Nov 2017.

[23] Z. Shi, Q. Huang, G. Wu, Y. Xu, M. Yang, X. Liu, C. Wang, and J. Ma, "Design and development of a tachometer using magnetoelectric composite as magnetic field sensor," IEEE Trans. Magn., vol. 54, no. 7, pp. 1-4, July 2018.

[24] K. Malleron, H. Talleb, A. Gensbittel, and Z. Ren, "Finite-element modeling of magnetoelectric energy transducers with interdigitated electrodes," IEEE Trans. Magn., vol. 53, no. 6, pp. 1-4, June 2017.

[25] T. Henneron and S. Clénet, "Application of the proper generalized decomposition to solve magnetoelectric problem," IEEE Trans. Magn., vol. 54, no. 3, pp. 1-4, March 2018 .

[26] T. A. Do, H. Talleb, A. Gensbittel, and Z. Ren, "3-d finite element analysis of magnetoelectric composites accounting for material nonlinearity and eddy currents," IEEE Trans. Magn., vol. 55, no. 10, pp. 1-8, Oct 2019.

[27] R. Orrú, R. Licheri, A. M. Locci, A. Cincotti, and G. Cao, "Consolidation/synthesis of materials by electric current activated/assisted sintering," Mater. Sci. Eng. R-Reports, vol. 63, no. 4-6, pp. 127-287, 2009.

[28] A. Aubert, V. Loyau, F. Mazaleyrat, and M. LoBue, "Uniaxial anisotropy and enhanced magnetostriction of CoFe2O4 induced by reaction under uniaxial pressure with SPS," J. Eur. Ceram. Soc., vol. 37, no. 9, pp. 3101 - 3105, 2017. [Online]. Available: http://www.sciencedirect.com/science/article/pii/S095522191730184X

[29] V. Loyau, V. Morin, G. Chaplier, M. LoBue, and F. Mazaleyrat, "Magnetoelectric effect in layered ferrite/PZT composites. Study of the demagnetizing effect on the magnetoelectric behavior," J. Appl. Phys., vol. 117, no. 18, 2015. [Online]. Available: http://scitation.aip.org/content/aip/journal/jap/117/18/10.1063/1.4919722

[30] A. Aubert, V. Loyau, F. Mazaleyrat, and M. LoBue, "Enhancement of the magnetoelectric effect in multiferroic CoFe2O4/PZT bilayer by induced uniaxial magnetic anisotropy," IEEE Trans. Magn., vol. 53, no. 11, pp. 1-5, Nov 2017. [Online]. Available: https://doi.org/10.1109/TMAG.2017.2696162

[31] D. Burdin, D. Chashin, N. Ekonomov, L. Fetisov, Y. Fetisov, G. Sreenivasulu, and G. Srinivasan, "Nonlinear magneto-electric effects in ferromagnetic-piezoelectric composites," J. Magn. Magn. Mater, vol. 358-359, no. Supplement C, pp. 98 - 104 , 2014. [Online]. Available: http://www.sciencedirect.com/science/article/pii/S0304885314000730

[32] D. Burdin, D. Chashin, N. Ekonomov, and Y. Fetisov, "Static deformation of a ferromagnet in alternating magnetic field," J. Magn. Magn. Mater., vol. 406, no. Supplement C, pp. 217 - 220, 2016. [Online]. Available: http://www.sciencedirect.com/science/article/pii/S0304885315309434

[33] G. Srinivasan, E. T. Rasmussen, and R. Hayes, "Magnetoelectric effects in ferrite-lead zirconate titanate layered composites: The influence of zinc substitution in ferrites," Phys. Rev. B, vol. 67, p. 014418, Jan 2003. [Online]. Available: http://link.aps.org/doi/10.1103/PhysRevB.67.014418

[34] X. Zhuang, C. M. Leung, G. Sreenivasulu, M. Gao, J. Zhang, G. Srinivasan, J. Li, and D. Viehland, "Upper limit for power conversion in magnetoelectric gyrators," Appl. Phys. Lett., vol. 111, no. 16, p. 163902, 2017. [Online]. Available: http://dx.doi.org/10.1063/1.5001165 